\title{
Comparison of Regression Model and Artificial Neural Network Model for the Prediction of Volume Percent of Diamond Deposition in Ni-Diamond Composite Coating
}

\author{
K. Ramanathan ${ }^{1, *}$, V.M. Periasamy ${ }^{2}$, U. Natarajan ${ }^{1}$ \\ ${ }^{I}$ A.C. College of Engineering and Technology, Karaikudi 630 004, TN, India \\ ${ }^{2}$ B.S.A crescent Engineering College, Vandalur, Chennai 600 048, TN, India
}

Received $12^{\text {th }}$ January 2008; accepted $14^{\text {th }}$ March 2008

\begin{abstract}
Nickel-diamond composite coatings are produced by electro deposition using sedimentation techniques on mild steel substrate at various cathode current density, $\mathrm{pH}$ and temperature. Electro deposition was carried out from a conventional Watts bath. Natural diamond powder of 6-12 $\mu \mathrm{m}$ size was used in the study. The volume percent incorporation of diamond on the coated specimens was measured gravimetrically. Artificial Neural Network (ANN) and regression models (a mathematical model) were used to predict the volume percent incorporation of diamond in the Ni-diamond metal matrix. In this work, Volume fraction of diamond deposition (Vfd) was taken as response variable (output variable) and current density, $\mathrm{pH}$ and temperature were taken as input variables. The prediction of response variable was obtained with the help of empirical relation between the response variable and input variables using ANN and also through DOE. The predicted values of the responses by ANN and regression models were compared with the experimental values and their closeness with the experimental values was determined.
\end{abstract}

Keywords: Volume fraction of diamond deposition (Vfd), ANN, Regression models, Design of Experiments (DOE), Ni-diamond composite coating.

\section{Introduction}

Diamond and diamond related composite coatings are considered as super abrasive materials harder than other materials. Due to their superior tribological properties, these materials are industrially important. But it is very difficult to

\footnotetext{
* Corresponding author. E-mail address: kalirams@yahoo.com
} 
make any component using diamond powder alone and this has necessitated the composite coatings.

A number of works has been reported on diamond coated tool using various techniques such as PVD and CVD [1-2]. J. Paulo Davim [1] studied the machinability of MMCs with PCD tool CVD deposited diamond coated tools. M. Chen et al. [2] showed the advantage of deposited thin film diamond over the sintered polycrystalline diamond of cutting tools with complex geometrics such as drill. Many works have also been published on Ni-Diamond electro composite coating [3-7]. Malathy Pushpavanam et al. [8] investigated volume percent incorporation of diamond powder in the Ni-cobalt-diamond composite coating gravimetrically. But still good prediction techniques like mathematical modeling and soft computing techniques such as artificial neural network (ANN) are very much required to predict the volume percent incorporation of diamond ( $\mathrm{Vfd}$ ) in the Ni-diamond metal matrix, since diamond percentage influences the wear resistance, hardness and other tribological properties.

Neural networks are composed of highly interconnected, simple processing units which are inspired by neural process observed in human brain. Successful applications can be found in areas such as process engineering, process control and estimation, pattern recognition, fault detection and image analysis. A common theme of these applications is the ability of the ANN to learn complex input - output relationships. They require no clearly defined algorithm or theory rather they have property of acquiring knowledge through the presentation of examples.

In the present work, two prediction techniques, namely mathematical modeling and artificial neural network, were developed for the prediction of volume percent incorporation of diamond in the nickel-diamond composite coating. A comparison between ANN and regression model has shown that ANN has greater prediction accuracy.

\section{Prediction techniques \\ Design of experiments}

A scientific approach to planning of experiments must be incorporated in order to perform an experiment most effectively. Statistical design of experiments is the process of planning the experiments so that appropriate data could be collected which may be analysed by statistical methods resulting in valid and objective conclusions.

Planning of experiments was employed in order to fulfill the following requirements:

- to get the uniformly distributed over the whole range of controllable factors to be investigated;

- to reduce the total number of experiments;

- to establish a relationship between different input variables and the output parameters accurately within the selected range of investigation. 


\section{$3^{k}$ Factorial design for three factors}

$3^{\mathrm{k}}$ factorial design is the most widely used factorial design having three levels for each of ' $\mathrm{k}$ ' factors. The three levels of factors are referred to as low (-1), intermediate (0) and high (+1). If there are three factors under study and each factor is at three levels arranged in a factorial experiment, then this constitutes a $3^{3}$ factorial design. Each main effect has two degrees of freedom; each two-factor interaction has four degrees of freedom. If they are $n$ replicates, then there are (n $\times 3^{3}-1$ ) degrees of freedom and $3^{3}(n-1)$ degrees of freedom for error [9].

\section{Artificial Neural Network}

Artificial Neural Networks (ANN) are biologically inspired, that is, they are composed of elements that perform in a manner that is analogous to the most elementary functions of the biological neurons. ANN has a parallel distributed architecture with a large number of neurons and connections. Each connection points from one node to another and is associated with a weight. Artificial neural networks are characterized by their topology, weight vectors and activation function that are used in the hidden layers and output layer. In the present paper, multilayer perceptrons, with each layer consisting of a number of computing neurons, have been used. A perceptron is nothing but a computing unit or neuron. A multilayer perceptron trained with the back propagation algorithm may be viewed as a practical way of performing a non-linear input-output mapping of a general nature.

The activation function used in the both hidden layer and output layer is a nonlinear function, where as for the input layer, no activation function is used since no computation is involved in the input layer. All neurons in a layer are fully connected to neurons in the adjacent layers. Information flows from one layer to other layer in a feed forward manner. The feed forward back propagation network is a popular architecture among different types of neural networks and finds applications in several areas of Engineering. The activation function $f(X)$ is a non-linear function and is given by

$$
f(X)=\frac{1}{1+\exp (-X)}
$$

where $f(X)$ is differentiable.

$$
X=\sum_{i=1} \text { WijUi }+ \text { threshold }
$$

Therefore, output of a neuron in the successive layer is given by

$$
\text { output, } \quad Y_{1}=\frac{1}{1+\exp \left[-\left(\sum_{i=1} W i j U i+\text { threshold }\right)\right]}
$$

where $W$ is the weight; $f(X)$ is the activation function; $U$ is the input node; $i$ and $j$ are number of input and hidden nodes, respectively. 
In the current application, the objective was to use the network to learn mapping between input and output patterns. The components of the input pattern consisted of the control variables cathode current density, $\mathrm{pH}$ and temperature, whereas the output pattern components represented the measured factor (volume percent of diamond of incorporation). The nodes in the hidden layer were necessary to implement the non-linear mapping between the input and output pattern.

In the present work, a method known as the active learning has been used. During the training process, initially all patterns in the training set were presented to the network and the corresponding error parameter (sum of squared errors over the neurons in the output layer) was found for each of them. Then the pattern with the maximum error was found which was used for changing the synaptic weights. Once the weights were changed, all the training patterns were again fed to the network and the pattern with the maximum error was then found. This process was continued till the maximum error in the training set became less than the allowable error specified by the user. This method has the advantage of avoiding a large number of computations, as only the pattern with the maximum error was used for changing the weights. This method could also avoid the problem known as thrashing (when weights were changed using a particular pattern may change to a higher value than the allowable value). It is proposed in the literature [10] that a multilayer perceptron trained with the back propagation algorithm may learn faster when the sigmoidal activation function used was asymmetric than when it was non-asymmetric.

\section{Experimental setup}

Experimental setup for plating is shown in Fig. 1. The plating was carried out in conventional watts bath using sedimentation techniques.

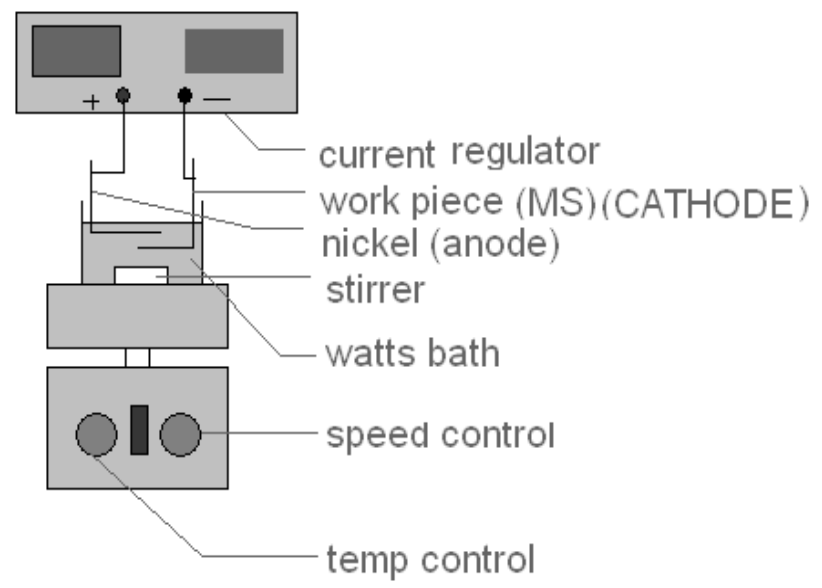

Figure 1. Experimental setup for Ni-diamond composite coating.

For the prediction of volume percent incorporation diamond under a variation of coating conditions, a training database with regard to different coating parameters needs to be established. A number of Ni-diamond composite coatings were carried out on mild steel substrate. 
Ranges of coating parameters in the coating process are as follows: current density, $\mathrm{i}=1-3 \mathrm{~A} / \mathrm{dm}^{2} ; \mathrm{pH}$ value $=2.5-4.5$; and temperature, $\mathrm{T}=30-60{ }^{\circ} \mathrm{C}$. For the above combination of parameters, twenty seven number of Ni-diamond composite coatings were obtained and their volume fraction of diamond incorporation were measured gravimetrically. The level designation of different process variables is shown in Table 1.

Table 1. Level designation of different process (coating) variables.

\begin{tabular}{|c|c|c|c|}
\hline Level & $\mathrm{i}\left(\mathrm{A} / \mathrm{dm}^{2}\right)$ & $\mathrm{pH}$ & $\mathrm{T}\left({ }^{\circ} \mathrm{C}\right)$ \\
\hline-1 & 1 & 2.5 & 30 \\
0 & 2 & 3.5 & 45 \\
1 & 3 & 4.5 & 60 \\
\hline
\end{tabular}

\section{Training the artificial neural network}

Artificial neural network consists of a number of layers (generally three) each with a number of nodes. These nodes process the data and pass it to the next layer. The input is given to the input layer and the network, after processing it, gives the result via the output layer. It has been found that they have the capacity of learning a complex polynomial equation of any degree. Since the working of the network is based on simple mathematical equations, they have a low response time which is extremely useful in controlling dynamic processes.

The neural network has to be first trained and then tested to use for application. The training was done off-line using a computer. The features current density, $\mathrm{pH}$ and temperature are the inputs and the volume percent incorporation of diamond is the output for training the neural networks. Weights between input layer and hidden layer and weights between hidden layer and the output layer are generated randomly for the selected topology of the network. The number of patterns used for the training of artificial neural network using feed forward back propagation algorithm is 27 . The learning parameter $(\eta)$ was 0.5 . Training of the network was stopped when the error reaches 0.001. The patterns are selected for training and testing the ANN. These selected patterns were normalized so that they lie between 0 and 1 . Twenty seven patterns were selected for training the ANN.

The inputs and outputs are normalized by

$$
X_{i}=\frac{X_{i}}{X_{\max }}
$$

where $X_{i}$ is the value of a feature and $X_{\max }$ is the maximum value of the feature. 


\section{Experimental results and discussion}

\section{Design of experiments}

A $3^{3}$ full factorial design was used in order to get the output data uniformly distributed all over the ranges of the input parameters. In this way 27 experiments were carried out with different combinations of the levels of the input parameters. Regression equations, to get the relation between different response variable (volume percent incorporation of diamond) and the input parameters (current density, $\mathrm{pH}$ and temperature) were found using software for statistical analysis, called SPSS - eq. (3). The squared residual value $\left(\mathrm{R}^{2}\right)$ for the regression model is found to be 0.8 .

$$
\mathbf{V f d}=1003.181 \mathrm{pH}^{0.0043} \mathrm{i}^{-0.041} \mathrm{~T}^{-0.919}
$$

\section{Artificial Neural Network}

The same data were fed to the artificial neural network program as training set of data. These experiments were called the training set of experiments. Training of the ANN was performed with an allowable error of 0.001 .

Once the network was trained such that the maximum error for any of the training data was less than allowable error, the weights and the threshold values were automatically saved by the program. As the input values from the validation experiments were given to the ANN program, the program predicted the required output. To validate the results of the regression equations and artificial neural network analysis 10 data from training sets were used.

Table 2. Experimental coating parameters, volume percent incorporation of diamond for verification.

\begin{tabular}{|c|c|c|c|c|}
\hline Test No & $\mathrm{pH}$ & $\mathrm{i} /\left(\mathrm{A} / \mathrm{dm}^{2}\right)$ & $\mathrm{T} /{ }^{\circ} \mathrm{C}$ & $\mathrm{Vfd}(\%)$ \\
\hline 1 & 2.5 & 1 & 30 & 42.9 \\
2 & 2.5 & 3 & 30 & 35.35 \\
3 & 3.5 & 3 & 30 & 43.23 \\
4 & 4.5 & 3 & 30 & 37.26 \\
5 & 3.5 & 1 & 45 & 34.99 \\
6 & 3.5 & 3 & 45 & 32.07 \\
7 & 4.5 & 3 & 45 & 24.44 \\
8 & 2.5 & 2 & 60 & 23.94 \\
9 & 3.5 & 2 & 60 & 30.3 \\
10 & 4.5 & 2 & 60 & 21.83 \\
\hline
\end{tabular}


To evaluate the developed networks for predicting volume percent incorporation of diamond, ten data as shown in Table 2 were used. Once the $\mathrm{pH}$, current density and temperature are fed into the predicted networks, the volume percent incorporation of diamond could be calculated quickly using the regression equations and ANN program.

A comparison of measured volume percent incorporation of diamond and predicted volume percent incorporation of diamond using the prediction techniques Regression model and Artificial Neural Network (ANN) is presented in the Table 3.

Table 3. Comparison of measured and predicted volume percent incorporation of diamond using DOE and ANN.

\begin{tabular}{|c|c|c|c|c|c|}
\hline \multirow{3}{*}{$\begin{array}{c}\text { Test } \\
\text { No. }\end{array}$} & \multicolumn{3}{|c|}{ Vfd (\%) } & \multicolumn{2}{c|}{ Error \% } \\
\cline { 2 - 6 } & \multirow{2}{*}{ Measured } & \multicolumn{2}{|c|}{ Prediction technique } & Prediction technique \\
\cline { 3 - 6 } & & DOE & ANN & DOE & ANN \\
\hline 1 & 42.9 & 44.13 & 42.92 & -2.87 & -0.046 \\
2 & 35.35 & 42.18 & 35.35 & -19.3 & 0 \\
3 & 43.23 & 42.25 & 43.24 & +2.27 & -0.023 \\
4 & 37.26 & 42.29 & 37.27 & -13.5 & -0.027 \\
5 & 34.99 & 30.44 & 34.98 & +13.0 & 0.028 \\
6 & 32.07 & 29.1 & 32.05 & +9.26 & 0.062 \\
7 & 24.44 & 29.1 & 24.42 & -19.1 & 0.812 \\
8 & 23.94 & 22.67 & 23.94 & +5.3 & 0 \\
9 & 30.3 & 22.71 & 30.29 & +25.0 & 0.033 \\
10 & 21.83 & 22.73 & 21.81 & -4.12 & 0.092 \\
\hline
\end{tabular}

The average absolute error between the measured volume percent incorporation of diamond and the predicted volume percent incorporation of diamond using the Regression model and ANN models are $0.4 \%$ and $0.0223 \%$, respectively, and it is shown in Figure 2.

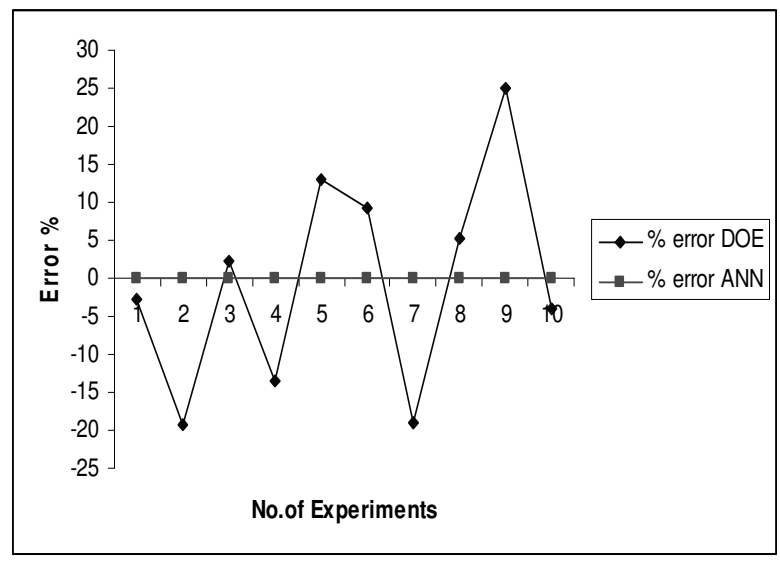

Figure 2. Comparison of errors in the prediction of volume percent incorporation of diamond. 
It is shown clearly that the prediction technique $\mathrm{ANN}$ is better than the regression analysis in predicting the volume percent incorporation of diamond in the nickeldiamond metal matrix.

\section{Conclusion}

The coating process variables were predicted using regression analysis and the artificial neural network models and the values obtained by both the methods were compared with the experimental values of the response variables to decide about the nearness of the predictions with the experimental values.

Within the range of input variables for the present case $(\mathrm{pH}=2.5$ to 4.5; current density (i) $=1$ to $3 \mathrm{~A} / \mathrm{dm}^{2}$; temperature $(\mathrm{T})=30$ to $60^{\circ} \mathrm{C}$ ), the results showed that artificial neural network comes ahead of the design of experiment in nearness of the predictions to the experimental values of volume percent incorporation of diamond as the average errors in case of ANN are less than that obtained using regression model.

\section{Acknowledgement}

The authors wish to express their gratitude to Dr. Malathy Pushpavanam, Deputy Director, Industrial Metal Finishing (IMF) Division, Central Electro Chemical Research Institute (CECRI), Karaikudi, who helped significantly in executing the electroplating and for making available laboratory facilities for the experiment.

\section{References}

1. J. P. Davim, J. Mater. Processing Technol. 128 (2002) 100-105.

2. M. Chen, X.G. Jain, F.H. Sun, B. Hu, X.S. Liu, J. Mater. Processing Technol. 129 (2002) 85.

3. M. Pushpavanam, Metal Finishing 95 (1997) 22

4. G. Sheela, M. Pushpavanam, Metal Finishing 100 (2002) 45.

5. $\quad$ K. Sato, K. Suzuki, Metal Finishing 72 (1984) 81.

6. E.C. Lee, J.W. Choi, Surf. Coatings Techn. 148 (2001) 234.

7. Y. Sofer, Y. Yarnitzky, S.F. Dirnfeld, Surf. Coatings Techn. 42 (1990) 227.

8. M. Pushpavanam, H. Manikandan, K. Ramanathan, Surface Coatings and Technology 201 (2007) 6372-6379.

9. D.C. Montgomery, Design and Analysis of Experiments, John Wiley and Sons, New York, 1959.

10. S. Haykin, Neural Networks - A Comprehensive Foundation, Macmillan College Publishing Co., New York, 1994. 